Pacific Journal of Mathematics

ABELIAN AND NILPOTENT SUBGROUPS OF MAXIMAL 


\title{
ABELIAN AND NILPOTENT SUBGROUPS OF MAXIMAL ORDER OF GROUPS OF ODD ORDER
}

\author{
ZVI ARAD
}

Denote the maximum of the orders of all nilpotent subgroups $A$ of class at most $c$, of a finite group $G$, by $d_{c}(G)$. Let $A_{c}(G)$ be the set of all nilpotent subgroups of class at most $c$ and having order $d_{o}(G)$ in $G$. Let $A_{\infty}(G)$ denote the set of all nilpotent subgroups of maximal order of a group $G$.

The aim of this paper is to investigate the set $A_{\infty}(G)$ of groups $G$ of odd order and the structure of the groups $G$ with the property $A_{2}(G) \subseteq A_{\infty}(G)$. Theorem 1 gives an expression for the number of elements in $A_{\infty}(G)$. Theorem 2 gives criteria for the nilpotency of groups of odd order.

In this paper $G$ is a finite group, and $\pi$ is a set of primes. If $G$ is of odd order, then $G$ is solvable [6].

1. Introduction. Denote the maximum of the orders of all nilpotent subgroups $A$ of class at most $c$, of a finite group $G$, by $d_{c}(G)$. Let $A_{c}(G)$ be the set of all nilpotent subgroups of class at most $c$ and having order $d_{c}(G)$ in $G$. Then $J_{c}(G)$ is the subgroup of $G$ generated by $A_{c}(G)$. In particular, $J_{1}(G)=J(G)$ is the Thompson subgroup of $G$. Moreover, $A_{x}(G)$ is the set of all nilpotnet subgroups of maximal order of a group $G$. Here $J_{\infty}(G)$ is the subgroup of $G$ generated by the elements of $A_{\infty}(G)$.

In this paper $G$ is a finite group, and $\pi$ is a set of primes. If $G$ is of odd order, then $G$ is solvable [6].

The aim of this paper is to investigate the set $A_{\infty}(G)$ for groups $G$ of odd order and the structure of the groups $G$ with the property $A_{2}(G) \subseteq$ $A_{x}(G)$.

We shall give, in Theorem 1, an expression for the number of elements in $A_{\infty}(G)$. In Theorem 2 we shall state criteria for the nilpotency of groups of odd order.

For groups $G$ with the property $A_{2}(G) \subseteq A_{\infty}(G)$, we have the following:

THEOREM 3. Let $G$ be a $\pi$-solvable group with an $S_{\pi}$-subgroup $K$ of G. Assume that $O_{\pi^{\prime}}(G)=1$ and that $A \in A_{2}(K) \cap A_{\infty}(K) \neq \varnothing$, then 
(i) If either 2, $3 \notin \pi$ or $O_{2}(A)$ is Abelian, then $O_{p}(A)=O_{p}(G)=$ $O_{p}(K)$, for every $p \in \pi$.

(ii) If $F(G)$ is odd and if $G$ has an Abelian $S_{2}$-subgroup, then $J_{\infty}(K)=J_{\infty}(G)=F(G)=F(K)=A$.

Three corollaries of Theorem 3 and further information can be found in Chapter 2.

Our notation is standard and is taken mainly from [8]. In particular, $\pi(G)$ will designate the set of primes dividing $|G|$ and $G_{\pi}$ denotes an $S_{\pi}$-subgroup of $G$. For the definitions of Sylow system, system normalizers and Carter subgroups of a group $G$ see [11], Definition $11.1 \mathrm{p}$. 726 and Definition 12.1 p. 736.

2. Statements and proofs of the main theorems. The next result is needed for the proofs of the main Theorems.

Proposition 1. Suppose $G$ is a group. Assume that A normalizes a nilpotent subgroup $B$ of $G$, and assume that at least one of the following conditions holds:

(i) $A \in A_{1}(G)$, and $B$ is Abelian ([3], Proposition 1).

(ii) $A \in A_{1}(G),|A|$ is odd, and an $S_{2}$-subgroup of $B$ is Abelian ([3], Proposition 1).

(iii) $A \in A_{2}(G)$ ([7]).

(iv) $A \in A_{c}(G), c \geqq 2,|B|$ is odd, and an $S_{2}$-subgroup of $A$ is Abelian ([4]). ([4]).

(v) $A \in A_{\infty}(G),|B|$ is odd and an $S_{2}$-subgroup of $A$ is Abelian

Then $A B$ is nilpotent.

Define $A_{\times}^{p}(G)$ to be the set $\left\{A_{p} / A \in A_{x}(G)\right\}$ of distinct $p$-subgroups of a group $G$, where $p$ is a prime.

THEOREM 1. Let $G$ be a group of odd order. Then

(i) $\mid\left(A_{x}(G)\left|=\left[G: N_{G}(A)\right]=\prod_{p \in \pi(A)}\right| A_{x}^{p}(G) \mid\right.$, where $A \in A_{x}(G)$.

(ii) $\left|A_{\propto}^{p}(G)\right| \equiv 1(\bmod p)$

(iii) $\left|A_{\propto}^{p}(G)\right| / h_{p}$, where $h_{p}=\left[G: N_{G}\left(G_{p}\right)\right]$

(iv) $G=\left\langle N_{G}(A) / A \in A_{\times}(G)\right\rangle$.

(v) If $A \in A_{\infty}(G)$ and $A_{p} \subseteq G_{p}$ then there exists $x \in G$ such that $G_{p} \cap G_{p}^{x}=A_{p}$.

For a discussion of the number $h_{p}$ see [9], Theorem 9.3.1.

Proof. Proposition 1(v) implies that every element of $A_{x}(G)$ con- 
tains $F(G)$. Therefore [13], Theorem 1 implies that $A_{\infty}(G)$ is a conjugate class. If $A, B \in A_{\infty}(G)$ then $\left[A_{p}, B_{q}\right]=1$ for every two distinct primes $p$ and $q$ by [11], Theorem 7.18, p. 705, proving (i). Let $A_{p} \subseteq G_{p}$. We shall prove that $N_{G}\left(G_{p}\right) \subseteq N_{G}\left(A_{p}\right)$. If $x \in N_{G}\left(G_{p}\right)$ then $\left\langle A_{p}^{x}, A_{p}\right\rangle \subseteq G_{p}$ is a $p$-group. Hence, $\left[\left\langle A_{p}^{x}, A_{p}\right\rangle, A_{p^{\prime}}\right]=1$. Therefore $\left\langle A_{p}^{x}, A_{p}\right\rangle A_{p^{\prime}}$ is nilpotent. Consequently, $A_{p}^{x}=A_{p}$, and $N_{G}\left(G_{p}\right) \subseteq N_{G}\left(A_{p}\right)$. Part (i) implies that $A_{\infty}^{p}(G)$ is a conjugate class. Hence, $\left|A_{\infty}^{p}(G)\right|=$ $\left[G: N_{G}\left(A_{p}\right)\right]$. By [14], Theorem 6.2.3, $\left|A_{\infty}^{p}(G)\right| \equiv 1(\bmod p)$. Thus (ii)-(iii) also hold. Let $\mathscr{F}=\left\{G_{p} / p \in \pi(G)\right\}$ be a sylow system of $G$. Let us denote the intersection of the normalizers of the subgroups of the given Sylow system by $N(\mathscr{F})$. By definition $N(\mathscr{F})$ is a system normalizer of $G$. Since $A_{\infty}(G)$ is a conjugate class of $G$ there exists $A(p) \in A_{\propto}(G)$ such that $[A(p)]_{p} \subseteq G_{p}$, for every $p \in \pi[A(p)]$.

As above $A=x[A(p)]_{p}$ is an element of $A_{\infty}(G)$, moreover $N_{G}(A) \supseteq N(\mathscr{F})$. Since $G$ is generated by the set of system normalizers of $G$ [11], we obtain that $G=\left\langle N_{G}(A) / A \in A_{\star}(G)\right\rangle$, proving (iv). Proposition 1 implies that $A_{p}=O_{p}\left(N_{G}\left(A_{p}\right)\right)$. As mentioned before $G_{p} \subseteq$ $N_{G}\left(A_{p}\right)$. Therefore by Ito's theorem [12] there exists $x \in G$ such that $G_{p} \cap G_{p}^{x}=A_{p}$.

The author knows of no counterexample to the conjecture that if $G$ is an arbitrary group then $A_{\infty}(G)$ is a conjugate class.

Let $\phi$ be the class of finite solvable groups in which the system normalizers are Carter subgroups. $\phi$-groups are discussed in [5] and [11], pp. 743-751.

THEOREM 2. Let $G$ be a group of odd order. Then

(i) If $G=B C$, where $B \in A_{b}(G), C \in A_{c}(G), b \geqq 1, c \geqq 1$, then $G$ is nilpotent.

(ii) $G$ is nilpotent if and only if $G \in \phi$ and the Carter subgroups of $G$ are elements of $A_{c}(G)$, for some integer $c$.

Proof. (i) Proposition 1 implies that $B F(G)$ and $C F(G)$ are nilpotent. Therefore, Theorem 1(i) and [13], Theorem 1 imply that there exist $x \in G$ and $A \in A_{x}(G)$ such that $B \subseteq A$ and $C \subseteq A^{x}$. Hence $G=$ $A A^{x}$. By [11], Theorem 7.18, p. $705 G$ is nilpotent.

(ii) Let $C$ be a Carter subgroup of $G$. Assume that $C \in A_{c}(G)$, for some integer $c$. Since $C F(G)$ is nilpotent by Proposition 1 , there exists $A \in A_{\infty}(G)$ such that $C \subseteq A$. Since $N_{G}(C)=C$, we obtain that $C=A$. By assumption $G \in \phi$. Therefore $A$ is a system normalizer. By the definition of system normalizer, Proposition 1 implies that $G$ is nilpotent. 
REMARK. Theorem 2(i) is true without assuming [6].

If $G=B C$, where $B \in A_{b}(G), C \in A_{c}(G), b \geqq 1, c \geqq 1$, then $G$ is solvable by [14], Theorem 13.2.9.

We shall say that $G$ is a $D_{\pi}$-group if all the maximal $\pi$-subgroups of $G$ are conjugate $S_{\pi}$-subgroups of $G$. If $G$ and every normal subgroup of $G$ is a $D_{\pi}$-group we will call $G$ a $D_{\pi^{-}}^{N}$ group.

Let $[A, B, C]$ denotes the triple commutator $[[A, B], C]$ of three subgroups $A, B, C$ of $G$. We say that $G$ is a $\pi$-stable group if it satisfies the following condition:

Let $K$ be an arbitrary $\pi$-subgroup of $G$. Let $A$ be an arbitrary $\pi$-subgroup of $N_{G}(K)$. Then if $[K, A, A]=1$, we have $A C_{G}(K) / C_{G}(K) \subseteq$ $O_{\pi}\left(N_{G}(K) / C_{G}(K)\right)$.

The next result is needed for the proof of Theorem 3 .

Proposition 2. Let $G$ be a $\pi$-stable $D_{\pi}^{N}$-group. Let $K$ be an $S_{\pi}$-subgroup of $G$. Assume that $A_{2}(K) \subseteq A_{\infty}(K), C_{G}(F(G) \subseteq F(G)$ and $O_{\pi^{\prime}}(G)=1$. Then we have

(i) $J_{2}(K) \operatorname{char} G$

(ii) If $|F(G)|$ is odd and $G$ has an Abelian $S_{2}$-subgroup, then $A_{2}(K)=A_{\infty}(K)$ and $J_{\infty}(K)=J_{\infty}(G)$.

Proof. Assume (i) is false for $G$. Let $\propto$ be an automorphism of $\dot{G}$, and choose $g \in G$ such that $K^{\alpha}=K^{8}$. If $J_{2}(K) \triangleleft G$, then

$$
\left(J_{2}(K)\right)^{\alpha}=J_{2}\left(K^{\propto}\right)=J_{2}\left(K^{g}\right)=\left(J_{2}(K)\right)^{g}=J_{2}(K) .
$$

Therefore $J_{2}(K)$ char $G$. Hence $J_{2}(K) \nless G$.

Let $L$ be the largest normal subgroup of $G$ which normalizes $J_{2}(K)$. Then $K \cap L$ is an $S_{\pi}$-subgroup of $L$ by [11], lemma 7.2 p. 444. Since $J_{2}(K \cap L)$ char $K \cap L$, it follows therefore, by a generalization of the Frattini argument that $G=L N$, where $N=N_{G}\left(J_{2}(K \cap L)\right)$. If $J_{2}(K) \subseteq K \cap L$, then $J_{2}(K)=J_{2}(K \cap L)$. In this case $N=N_{G}\left(J_{2}(K)\right)$. But then $G=L N \subseteq N_{G}\left(J_{2}(K)\right)$ and $J_{2}(K) \triangleleft G$, a contradiction. Thus, we may assume that $J_{2}(K) \not \subset L \cap K$. By Proposition $1, F(G) \subseteq A$ for every $A \in A_{2}(K) \subseteq A_{\infty}(K)$. In particular, $[F(G), A, A]=1$. Since $G$ is $\pi$ stable, it follows from the definition that $A C_{G}(F(G)) / C_{G}(F(G)) \subseteq$ $O_{\pi}\left(G / C_{G} F(G)\right)$. By definition of $L$ and by hypothesis $C_{G}(F(G) \subseteq$ $F(G) \subseteq L$. Hence $A L / L \subseteq O_{\pi}(G / L)$ for every $A \in A_{2}(K) \subseteq A_{\infty}(K)$.

However, we claim that $O_{\pi}(G / L)=1$. Indeed, set $O_{\pi}(G / L)=$ 
$T / L . K \cap T$ is an $S_{\pi}$-subgroup of $T$, therefore $(K \cap T) L / L$ is an $S_{\pi}$-subgroup of $T / L$ by [11], Lemma 7.2 p. 444 . Therefore $(K \cap T) L=$ $T$. But $K \cap T \subseteq N_{G}\left(J_{2}(K)\right)$, thus $K \cap T \subseteq L$, whence $T=L$, and $O_{\pi}(G / L)=1$. Therefore $A \subseteq L$ for every $A \in A_{2}(K) \subseteq A_{\infty}(K)$. Therefore $J_{2}(K) \subseteq L \cap K$, a contradiction. Therefore $J_{2}(K)$ char $G$, proving (i).

Clearly $F(G) \subseteq F(K)$ and $|F(K)|$ is odd, since $C_{G}(F(G) \subseteq F(G)$. Hence by hypothesis $C_{G}(F(K)) \subseteq F(K)$. Proposition 1 yields that $F(K) \subseteq A$ for every $A \in A_{\infty}(K)$. Therefore $A_{\infty}(K)=A_{2}(K)$ as a consequence of [13], Theorem 1. Proposition 1 implies that $F(G) \subseteq A$ for every $A \in A_{\infty}(G)$. Therefore $Z(A) \subseteq C_{G}(F(G))=Z(F(G))$. By hypothesis $F(G)$ is a $\pi$-group. Hence $A$ is a $\pi$-group for every $A \in A_{\infty}(G)$. Since $G$ is a $D_{\pi}^{N}$-group and $J_{\infty}(K)$ char $G$ by part (i), we obtain:

$$
J_{\infty}(G)=\left\langle J_{\infty}\left(K^{X}\right) / X \in G\right\rangle=J_{\infty}(K)
$$

proving (ii).

We now obtain:

Proof of Theorem 3. (i) We use induction on the order of $G$. Let $T=O_{p}(G), \quad H=O_{p p^{\prime}}(G), \quad G^{*}=A H, \quad$ and $\quad K^{*}=A(K \cap H)$. Then $K \cap H$ is an $S_{\pi}$-subgroup of $H$ and $K^{*}$ is an $S_{\pi}$-subgroup of $G^{*}$.

Suppose that $G^{*} \subset G$. Since $A \subseteq K^{*}, A \in A_{x}\left(K^{*}\right)$. By induction, $O_{p}(A) \subseteq O_{p}\left(G^{*}\right)$. Hence

$$
\left[H, O_{p}(A)\right] \subseteq H \cap O_{p}\left(G^{*}\right) \subseteq O_{p}(H)=T .
$$

Therefore, $O_{p}(A) T / T \subseteq C_{G / T}(H / T) \subseteq H / T$, by [8], p. 228. Consequently, $O_{p}(A) \subseteq H$. So,

$$
O_{p}(A) \subseteq H \cap O_{p}\left(G^{*}\right)=O_{p}(H)=T .
$$

On the other hand, $T=O_{p}(G) \subseteq O_{p}(A)$ by Proposition 1. Therefore, $O_{p}(A)=O_{p}(G)$, as desired.

Suppose that $G^{*}=G$. Then $A_{p} T$ is an $S_{p}$-subgroup of $G$. By Proposition 1, $T=O_{p}(G) \subseteq O_{p}(A)$. Therefore $O_{p}(A)$ is an $S_{p}$-subgroup of $G$. It is well known that $G$ is $p$-strongly solvable for every $p \in \pi$ $\{2\}$. By definition $G$ is $p$-stable for $p=2$. Hence $G$ is $p$-stable for every $p \in \pi$. Therefore $G=O_{p^{\prime}, p . p^{\prime}}(G)$ by Proposition 2. Hence $O_{p}(A) \subseteq$ $O_{p^{\prime} p}(G)$. By Proposition 1, $A F\left(O_{p^{\prime}}(G)\right)$ is nilpotent. Hence $O_{p}(A) \subseteq$ $C_{G}\left(F\left(O_{p},(G)\right)\right)$. By [3], Lemma $4, O_{p}(A) \subseteq O_{p}(G)$, for every $p \in \pi$. Therefore $O_{p}(A)=O_{p}(G)$, as desired. In particular $O_{p}(A)=O_{p}(K)$, for every $p \in \pi$, proving (i). 
(ii) Define $\sigma=\pi(F(G))$. It is well known that $G$ is a $D_{\sigma^{-}}^{N}$ group. By [1], Corollary 4.8, $C_{G}(F(G)) \subseteq F(G)$. Proposition 1 yields that $F(G) \subseteq A$. Therefore $Z(A) \subseteq C_{G}(F(G)) \subseteq F(G)$. Hence $A$ is a $\sigma$-group. By definition $O_{\sigma}(G)=1$. Since by hypothesis $G$ is $p$ strongly solvable, for every $p \in \sigma, G$ is $\sigma$-stable by [2], Lemma 3.4. Let $R$ be an $S_{\sigma}$-subgroup of $G$. Then $J_{\infty}(R)=J_{\infty}(K)=J_{\infty}(G)$ by Proposition 2. So $J_{\infty}(K)=J_{\infty}(G)=F(G)=F(K)=A$, by part (i).

The author knows of no counterexample to the conjecture that if $G$ is a group of odd order then $O_{p}\left(A_{2}\right) \subseteq O_{p}(G)$, where $p \geqq 5$ and $A_{2} \in A_{2}(G)$.

Theorem 3 has three corollaries.

COROLlary 1. If the Sylow subgroups of a solvable group $G$ are all Abelian or if $G$ is of odd order and the Sylow subgroups of $G$ are of class at most 2, then $F(G) \in A_{\infty}(G)$.

Corollary 2. If $P$ is an $S_{p}$-subgroup of a group $G, p$ odd, $\operatorname{cl}(P) \leqq 2$, and if $N_{G}(P)$ has a normal p-complement, then so does $G$.

Proof. Following the method of [8], Theorem 8.3.1 and using Theorem 3 we obtain Corollary 3 . One must take $N_{G}(P)$ instead of $N_{G}(Z J(P))$ in the above mentioned theorem and its proof.

Corollary 2 is a known result. It can be obtained by [11], Theorem 8.1 p. 447.

We shall say that $G$ is a $\psi$-group if $A_{\star}(G)$ and Carter subgroups of $G$ coincide.

Corollary 3. Let $G$ be a group of odd order. Assume that $G$ and every subgroup of $G$ is a $\psi$-group, then $G$ is nilpotent.

Proof. Let $G$ be a minimal counterexample. By induction every proper subgroup of $G$ is nilpotent. Therefore the Sylow subgroups of $G$ are of class at most 2 by [11], Theorem 5.2, p. 281. Hence, Theorem 3 implies that $\left\langle A_{\star}(G)\right\rangle=F(G)$ is a Carter subgroup of $G$. Therefore $G$ is nilpotent as desired.

\section{REFERENCES}

1. Z. Arad, Products of fitting classes of finite groups, Israel J. Math., 12 (1972), 61-69.

2. - A characteristic subgroup of $\pi$-stable groups, Canad. J. Math., XXVI, 6 (1974), $1509-1514$.

3. Z. Arad and G. Glauberman, A characteristic subgroup of a group of odd order, Pacific J. Math., 56 (1975), 305-319. 
4. A. Bialostocki, On products of two nilpotent subgroups of a finite group. Israel J. Math., 20 (1975), 178-188.

5. R. W. Carter, On a class of finite solvable groups, Proc. London Math. Soc., (3) 9 (1959), 623-640.

6. W. Feit and J. G. Thomposon, Solvability of groups of odd order, Pacific J. Math., 13 (1963), 775-1029.

7. G. Glauberman, On Burnside's other $p^{a} q^{b}$ Theorem, Pacific J. Math., 56 (1975), 469-476.

8. D. Gorenstein, Finite Groups, Harper and Row, New York, (1968).

9. M. Hall, The Theory of Groups, New York: Macmillan Company (1959).

10. P. Hall, On the system normalizers of a solvable group, Proc. London Math. Soc., (2) 43 (1937), 507-508.

11. B. Huppert, Endlich Gruppen I, Springer-Verlag, New York (1967).

12. N. Ito, Über der Kleinsten p-Durchshnitt auflosbarer Gruppen, Arch. Math., 9 (1958), 27-32.

13. A. Mann, Injectors and normal subgroups of finite groups, Israel J. Math., 9, No. 4, (1971), 554-558.

14. W. R. Scott, Group Theory, Englewood Cliffs (New-Jersey) Prentice-Hall, Inc. (1964).

Received September 26, 1974 and in revised form October 30, 1975.

BAR-ILAN UNIVERSITY 




\section{Pacific Journal of Mathematics}

\section{Vol. 62, No. $1 \quad$ January, 1976}

Mieczyslaw Altman, Contractor directions, directional contractors and

directional contractions for solving equations . .................. 1

Michael Peter Anderson, Subgroups of finite index in profinite groups .........

Zvi Arad, Abelian and nilpotent subgroups of maximal order of groups of odd order

John David Baildon and Ruth Silverman, On starshaped sets and Helly-type theorems ..........................................

John W. Baker and R. C. Lacher, Some mappings which do not admit an

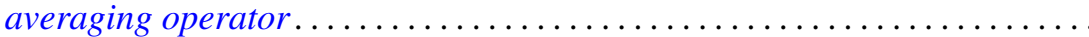

Joseph Barback, Composite numbers and prime regressive isols . . . . . . . . . .

David M. Boyd, Composition operators on $H^{p}(A) \ldots \ldots \ldots \ldots \ldots \ldots \ldots$

Maurice Chacron, Co-radical extension of PI rings . . . . . . . . . . . . .

Fred D. Crary, Some new engulfing theorems . . . . . . . . . . . . . . .

Victor Dannon and Dany Leviatan, A representation theorem for convolution transform with determining function in $L^{p} \ldots \ldots \ldots \ldots \ldots \ldots \ldots \ldots \ldots \ldots \ldots \ldots \ldots \ldots$

Mahlon M. Day, Lumpy subsets in left-amenable locally compact

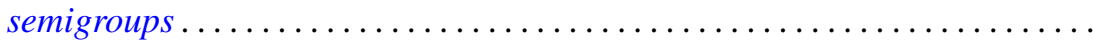

Michael A. Gauger, Some remarks on the center of the universal enveloping algebra of a classical simple Lie algebra . .

David K. Haley, Equational compactness and compact topologies in rings

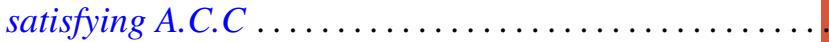

Raymond Heitmann, Generating ideals in Prüfer domains .

Gerald Norman Hile, Entire solutions of linear elliptic equations with

Laplacian principal part. .

Richard Oscar Hill, Moore-Postnikov towers for fibrations in which $\pi_{1}$ (fiber) is non-abelian

John Rast Hubbard, Approximation of compact homogeneous maps . .

Russell L. Merris, Relations among generalized matrix functions . .

V. S. Ramamurthi and Edgar Andrews Rutter, On cotorsion radicals ...

Ralph Tyrrell Rockafellar and Roger Jean-Baptiste Robert Wets, Stochastic

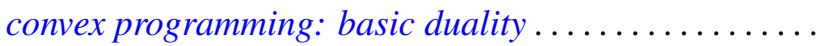

Alban J. Roques, Local evolution systems in general Banach spaces ..

I. Bert Russak, An indirect sufficiency proof for problems with bounded state variables.

Richard Alexander Sanerib, Jr., Ultrafilters and the basis property. .

H. A. Seid, The decomposition of multiplication operators on $L_{p}$-spaces . .

Franklin D. Tall, The density topology .................. 